\title{
Mycobacterium ulcerans Infection in French Guiana; Current State of Knowledge
}

\author{
Pierre Couppié, Romain Blaizot, Camilla J. Velvin, \\ Maylis Douine, Marine Combe, Mathieu Nacher, \\ and Rodolphe E. Gozlan
}

\section{Introduction}

M. ulcerans infections (Buruli ulcer; BU) have been reported in French Guiana since the 1960s [1]. It is striking to see that French Guiana concentrates most of the cases of BU in America with only a few cases reported in Suriname, Peru, Mexico and Bolivia in the past $[2,3]$. Either BU is underdiagnosed and underreported in most of the continent; or the specific environmental conditions in French Guiana represent a unique niche for the disease to appear; or a sublineage of $M$. ulcerans has established in French Guiana that has a higher virulence than other members of the ancestral lineage found in the Americas.

French Guiana is a tropical country located in South America on the Atlantic coast between Suriname and northern Brazil. It is mostly covered by Amazonian rainforest with the exception of the coastal strip, which is mainly composed of marshy savannah and mangroves. Most inhabitants reside along the coastal strip. There are several communities living in French Guiana, including Creoles, Maroons, Amerindians, mainland French people, Asians and many immigrants from South America and the Caribbean Islands. Since the early 1970s, French Guiana has seen

P. Couppié $(\bowtie) \cdot$ R. Blaizot

Service de dermatologie, Cayenne Hospital, Cayenne Cedex, French Guiana

Université de Guyane, EA3593 Ecosystèmes Amazoniens et Pathologie Tropicale, Cayenne, French Guiana

e-mail: pierre.couppie@ch-cayenne.fr

C. J. Velvin · M. Combe · R. E. Gozlan

UMR ISEM, Université de Montpellier, CNRS, IRD, EPHE, Montpellier, France

M. Douine $\cdot$ M. Nacher

Université de Guyane, EA3593 Ecosystèmes Amazoniens et Pathologie Tropicale,

Cayenne, French Guiana

Centre d'Investigation Clinique, CIC Inserm 1424, Cayenne Hospital,

Cayenne Cedex, French Guiana 
very rapid demographic and socioeconomic changes, with a population that increased from 45,000 in 1969 to 260,000 in 2017 and a gross domestic product (GDP) that grew by $7.2 \%$ per year between 1960 and 2002. Although the territory has developed rapidly, with improving living conditions such as better hygiene, gradual elimination of intestinal nematodes and easier access to health care, several migration waves from poor regions of Southern and Central America have led to pockets of poverty.

Here, we review current knowledge of BU in French Guiana with specific emphasis on links of $M$. ulcerans to the human host and the natural environment.

\section{$2 \quad$ Links Between the Human Host and the Bacteria}

\subsection{Risk Factors of BU in French Guiana}

A case-control study was conducted between 2002 and 2004 to determine relationships between the presence of $M$. ulcerans in the environment and human activities associated with the occurrence of BU in French Guiana [4]. The study involved 30 laboratory confirmed BU cases and 60 controls matched for age, gender and town of residence. Increased risk of contracting the disease was strongly associated with environmental factors and human behavioural factors. The environmental factors implicated in the transmission of M. ulcerans were proximity of the home or workplace of patients to natural freshwater habitats (e.g. marshes, rivers, and floodable areas). In addition, some behavioural factors such as hunting, fishing, recreational activity, and contact with river banks were associated with BU cases. These results are consistent with previous studies performed in Africa and Australia that have shown that people who are in close proximity to water sources or take part in outdoor activities are at higher risk of contracting BU. These findings suggest that informal professional or recreational activities like hunting or fishing and contact with those water sources are risk factors for contracting BU in French Guiana (Fig. 1).

\subsection{Epidemiology and Clinical Aspects of BU in French Guiana}

The epidemiology of BU in French Guiana since the first diagnosed case in 1969 was recently studied with the aim of characterising the incidence of the disease in the past 45 years, the temporal demographic trends of patients, and the associated clinical aspects of the disease [1]. Data were collected at Cayenne Hospital's dermatology department where 245 BU patients were reported between 1969 and 2013. Most cases occurred in coastal areas surrounded by marshy savannah. The highest number of cases was reported in Cayenne (47\% of diagnosed cases), followed by Mana (20\%), Sinnamary (16\%), Kourou (11\%), and forest areas (6\%). The annual number of cases varied widely, with no cases in some years and up to 27 in others. Over the 45 years, the mean annual number of new cases was 5.4 per year and the 


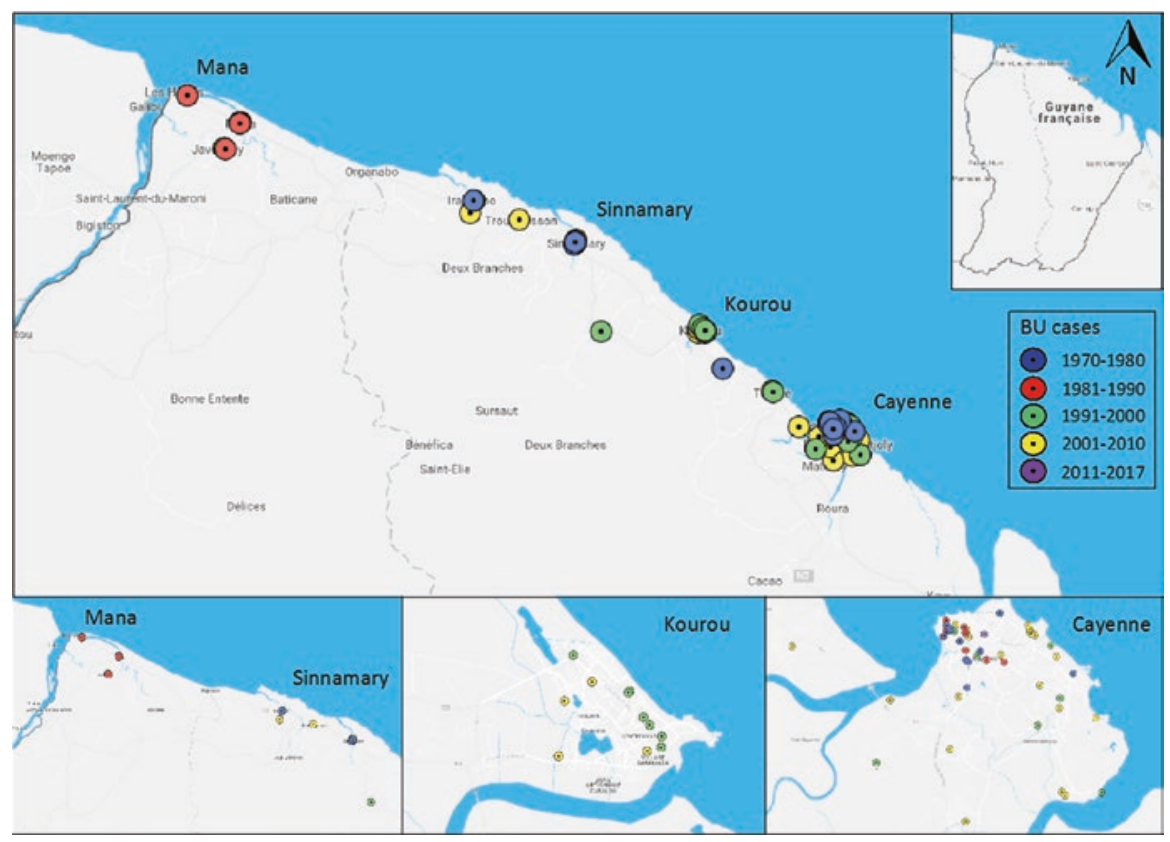

Fig. 1 Location of BU cases in French Guiana identified between 1969 and 2016. Precise geographic coordinates were available for 94 cases only. BU cases were sorted by periods of 10 years that are represented by different colors in the map. Top: location of the $94 \mathrm{BU}$ cases along the coastline. Bottom: zoom on the Mana and Sinnamary region, Kourou and Cayenne city. The map was created online with BatchGeo

mean annual incidence rate was 4.3 per 100,000 . However, the annual incidence of BU significantly decreased over time across all districts, from 6.1 infections per 100,000 persons in $1969-83$ to 3.5 infections per 100,000 persons in 1999-2013. The highest mean annual incidence rate over the 45 years was found in the western coastal area around Sinnamary (21.1 per 100,000 persons) and Mana (21.2 per 100,000 persons). Annual incidence peaks were observed in the Sinnamary area between 1974 and 1978 ( 71.8 cases per 100,000 persons) and another in the Mana area between 1984 and 1988 (101.4 cases per 100,000 persons). Over time, the mean age of patients with BU increased significantly in French Guiana between 1969 and 2013. The proportion of children diagnosed with BU before 1984 was similar to that in Africa (more than 70\%), but decreased with time, reaching around $21 \%$ in 1999-2013, which is closer to the proportion observed in Australia. Men and women were similarly affected in French Guiana, as observed in other countries and lesions were usually ulcerated (92\%) mainly with single ulcers with undermined edges. Nodules and plaques were less frequent, sometimes ulcerated. Only $8 \%$ of the patients had multiple lesions. Lesions mainly affected limbs (lower limbs in $66 \%$ of patients; upper limbs in $24 \%$ of patients), which is similar to settings in Africa and Australia. Adults were significantly more often affected on the ankles than were children (14\% vs $4 \%$ ). No bone infections were diagnosed in French 
Guiana whereas some studies in Africa reported osteomyelitis in up to $10 \%$ of cases. With the use of antibiotic therapy as first-line treatment of the infection since the 2000s in French Guiana (rifampicin combined with amikacin or clarithromycin), we described some cases of patients with a paradoxical reaction, an apparent worsening of the lesions during treatment [5]. These reactions are also described in Australia and Africa.

The global change of the epidemiology of BU in French Guiana over the past 45 years, (i.e. decrease in the incidence of BU and a decrease in the proportion of children with infections) might have been mostly driven by better living conditions (higher GDP per person, changes in clothing, improved hygiene, wound care with soap), prophylactic recommendations (improvement of BCG immunisation coverage), reduction of children coming into contact with freshwater habitats because of education and protection of water sites as well as access to health care (access to the French universal healthcare system with faster access to care). These data indicate an epidemiological transition from the African-like epidemiology, with mostly children infected, to the Australian-like epidemiology, with mostly adults infected. The absence of bone lesions in this patient series also raises the hypothesis of specific genetic variants of $M$. ulcerans in French Guiana.

Finally, some environmental changes induced by human activities, such as increased deforestation, building of dams (Sinnamary) and irrigation systems (Mana) that deeply modified the floodplain structure as well as modifications in rainfall patterns due to global climate change could also have influenced the change of BU incidence in French Guiana.

\subsection{A Link Between Rainfall and BU Cases}

Links between changes in rainfall and outbreaks of BU in French Guiana were studied from 1969-2012 [6]. During the study period, four inter-annual peaks in rainfall, each followed by three inter-annual periods of rainfall recessions were observed, with three corresponding peaks and recessions of BU cases. Intra-annual analysis showed bi-annual peaks in the number of new BU cases; after a peak in rainfall, the number of BU cases increased during a dry period. This was observed over several years but also annually, when BU cases are most likely to spike in the annual seasons. It has also been shown that climatic anomalies such as peaks in sea surface temperature (El Niño) create a decline in the oscillation of BU cases. This shows that outbreaks of BU in French Guiana can be triggered by combinations of rainfall patterns occurring on a long (i.e., several years) and short (i.e., seasonal) temporal scale, in addition to stochastic events driven by the El Niño-Southern Oscillation that may modulate these patterns.

Two hypotheses may explain the observed peaks and recessions of cases of BU. Firstly, the long periods of wet weather characterised by floods, during which the bacteria could be dispersed across the floodplain, are followed by a decrease in rainfall that leads the flooded areas to recede into a series of disconnected stagnant pools prone to bacterial development [7]. These rapid changes in aquatic habitat 
from flowing, well oxygenated habitats with rich animal communities to eutrophic, diversity poor communities have been shown to favour the development of M. ulcerans in the environment. Secondly, during the dry period these newly created freshwater pools become more easily accessible by foot for hunting or fishing or simply for recreational bathing. The combination of emergence of favourable habitats for M. ulcerans and increased contact of the local population with these habitats during dry periods could explain the observed link between rainfall and increased numbers of BU cases in French Guiana.

\subsection{Genetic Diversity of M. ulcerans Among Local BU Cases}

Only two clinical strains of M. ulcerans (ITM7922 and Mu_1G897) from French Guiana were genotyped prior to 2014 [8-10]. Comparative whole genome sequencing showed that the isolate Mu_1G897 belongs to the ancestral lineage of M. ulcerans. In the phylogenomic analysis it clustered with fish pathogens belonging to the sublineage 1 of the ancestral lineage, as defined by Doig et al. [8]. A recent study based on Multilocus Variable Number Tandem Repeat Analysis (MLVA) looked at the genetic diversity of $M$. ulcerans strains isolated from BU patients from French Guiana between 1994 and 2013 [11]. A total of 23 DNA samples obtained from 23 patients were purified from ulcer biopsies or derived from cultures. Three allelic combinations were characterised in this study: genotype I, which has been described previously (ITM7922 and Mu_1G897), genotype III, which is very similar to genotype I and genotype II which has distinctly different characteristics in comparison with the other two genotypes. In the MLVA based phylogenetic tree the classical lineage strains from Africa and Australia formed a separate cluster, indicating that all three genotypes from French Guiana belong to the ancestral lineage. This study revealed for the first time genetic variability between clinical isolates of $M$. ulcerans in French Guiana, a diversity that appears to be more pronounced than in Africa, where closely related local clonal complexes are observed. In addition, no correlations were observed between the MLVA-based phylogenetic clustering of the strains from French Guiana and the age of patients, date of infection or geographic location.

\section{$3 \quad$ Environment and the Bacteria}

\subsection{First Detection of Mycobacterium ulcerans DNA in Environmental Samples from French Guiana}

In French Guiana in 2014 a study identified for the first time the presence of $M$. ulcerans DNA in environmental samples from South America [12]. A total of 163 environmental samples, taken from 23 freshwater bodies were tested for the presence of the $M$. ulcerans-specific markers IS2404 and KR by real-time (q) PCR. Sampling sites were selected by looking for (1) water-bodies near the homes 
of BU cases with habitats representing a high probability of human contact (including paths near or through the water site, presence of fishing or boating activities or other recreational uses and close proximity to human settlements) and (2) similar habitats in areas where there were no BU cases and with little human contact. Samples taken included water (from various sampling sites including bank-side, centre of the water body, water from within aquatic vegetation, shaded areas and sun exposed areas), soil (underwater sediment), detritus, dominant aquatic and semi-aquatic plant species, algae and biofilms. In addition, abiotic data such as dissolved oxygen, $\mathrm{pH}$, conductivity and water temperature were measured.

Only five samples out of 163 were found positive for both IS2404 and KR in three different water bodies from the Sinnamary and Tonate districts. All three positive sites were typologically similar, with areas that were highly stagnant, forming shallow water bodies. There was no statistical significance for differences in abiotic data between positive and negative sites. These three freshwater bodies with qPCR positive samples were on floodplains, suggesting that in French Guiana, this type of environment constitutes a source for environmentally-persistent mycobacteria or a "receptacle" concentrating these bacilli from further upstream (Fig. 2).

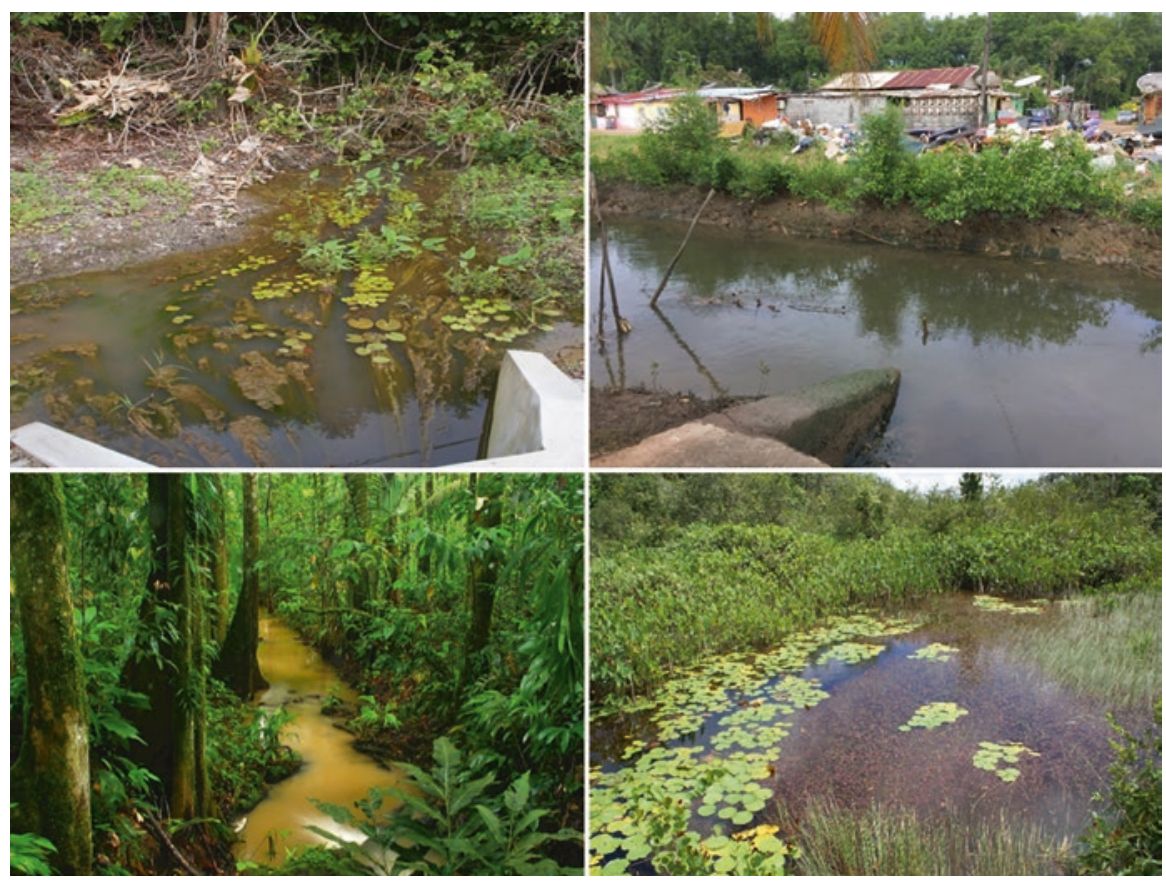

Fig. 2 Diversity of environmental sites positive for M. ulcerans DNA in French Guiana. For each site sediment, water and invertebrates samples were tested for M. ulcerans DNA by targeting the genetic markers IS2404 and KR [6] 


\subsection{Biodiversity Drivers of $M$. ulcerans Distribution Across Freshwater Habitats}

A study on a larger number of environmental samples was conducted to study the links between the drivers of freshwater foodwebs in French Guiana and the emergence of M. ulcerans in the environment. A collection of 3600 invertebrates and fish were sampled and catalogued from 17 freshwater sites across French Guiana [7, 13]. Landscape data as a measure of land use along a gradient from urban through agricultural to pristine rain forest were extracted from two maps including the CORINE 2006 European land cover map using satellite-derived data and the Hansen deforestation map. Each site was surveyed twice in the rainy season, during February and June 2013. Each insect taxon was positioned in the foodweb using a measure of stable isotopes (nitrogen $\delta^{15} \mathrm{~N}$ and carbon $\delta^{13} \mathrm{C}$ ).

For each site, the $M$. ulcerans load was calculated for each taxon from the qPCR data for both IS2404 and KR molecular markers. Each taxon's regional mean trophic niche width (metric of total available trophic niche space within a site), vulnerability (number of potential predator species for each prey taxon), connectance (a standard measure of foodweb complexity) and generality (number of potential prey species each predator taxon can consume) were calculated and weighted by the abundance at each site. The relationships between land cover data and trophic niche width were identified at the local site level using general additive models (GAMs) of the level of deforestation within each buffer zone in addition to urban and agricultural cover.

A total of 78 different freshwater taxa were identified, of which 383 specimens representing 44 taxa tested positive for both IS2404 and KR. The highest concentrations of $M$. ulcerans were in species lower in the food chain, suggesting a diet high in aquatic algae, detritus, diatoms and similar food sources. These taxa were predominantly invertebrates in both adult and larval form.

Deforestation and either agricultural or urban intrusion were associated with a decline in local trophic niche width, resulting in decreased regional means of vulnerability and generality of taxa. This had an important effect on the potential local M. ulcerans load. Host taxa, which on average carried a high level of M. ulcerans, were most abundant at sites where there was a very low level of vulnerability and a mid-level of generality.

\section{Conclusion}

The epidemiology of BU in French Guiana has changed in the past 45 years, mainly with respect to a decrease in incidence, an increase in the average age of patients and peak incidences in geographical areas that have undergone environmental changes. Improved socioeconomic conditions may explain this transition from an African-type epidemiology to an Australian-type epidemiology. In addition, a link between the occurrence of human cases and climatic patterns has clearly been established. The influence of rainfall has been evidenced on an intra-annual 
(6 months) and inter-annual (8 years) scale to which climatic anomalies such as El Niño are randomly added. Overall, episodes of high rainfall followed by relative drought are significantly associated with the increase in BU cases.

From a genetic perspective, the fairly large genetic diversity of $M$. ulcerans within French Guiana is rather unusual. The strains causing disease in French Guiana belong to the ancestral lineage and are markedly different from classical lineage strains from Africa and Australia. This seems to eliminate the possibility of an introduction of M. ulcerans into French Guiana with the arrival of populations from endemic areas of Africa. However, the diversity of strains present in the environment remains unknown and needs to be better understood in order to highlight potential selective processes shaping the genome of disease causing strains.

Current environmental data show a relatively high abundance of $M$. ulcerans DNA across freshwater habitats along the coastline of French Guiana. Humaninduced environmental modifications (e.g. deforestation, agriculture, urbanization) have been found to be significant drivers of foodweb changes towards an increase in taxa with higher $M$. ulcerans loads.

Ongoing studies are looking to further our understanding of transmission pathways from the environment to the local human population. There may be multiple pathways, which may act as strong selective filters for M. ulcerans strains found in the environment.

\section{References}

1. Douine M, Gozlan G, Nacher M, Dufour J, Reynaud Y, Elguero E, Combe M, Velvin CJ, Chevillon C, Berlioz-Arthaud A, Labbé S, Sainte-Marie D, Guégan JF, Pradinaud R, Couppié P (2017) Mycobacterium ulcerans infection (Buruli ulcer) in French Guiana, South America, 1969-2013: an epidemiological study. Lancet Planet Health 1:e65-e73

2. Guerra H, Palomino JC, Falconi E, Bravo F, Donaires N, Van Marck E, Portaels F (2008) Mycobacterium ulcerans disease, Peru. Emerg Infect Dis 14:373-377

3. Röltgen K, Pluschke G (2015) Epidemiology and disease burden of Buruli ulcer: a review. Res Rep Trop Med 6:59-73

4. Elguero E, Broutin H, Nacher M, Chevillon C, Guegan JF, Couppié P (2009) Environment risk factors of Buruli ulcer. A case-control study in French Guiana. In: Second International Conference on Buruli Ulcer, Cotonou, Benin, March 30-April 3, 2009

5. Sambourg E, Dufour J, Edouard S, Morris A, Mosnier E, Reynaud Y, Sainte-Marie D, Nacher M, Guégan JF, Couppié P (2014) Réponses et réactions paradoxales au cours du traitement médicamenteux de l'infection à Mycobacterium ulcerans (ulcère de Buruli). Quatre observations en Guyane française. Ann Dermatol Venereol 141:413-418

6. Morris A, Gozlan RE, Hassani H, Andreou D, Couppié P, Guégan JF (2014) Complex temporal climate signals drive the emergence of human water-borne disease. Emerg Microbes Infect 3:e56

7. Combe M, Velvin CJ, Morris A, Garchitorena A, Carolan K, Sanhueza D, Roche B, Couppié P, Guégan JF, Gozlan RE (2017) Global and local environmental changes as drivers of Buruli ulcer emergence. Emerg Microbes Infect 6:e22

8. Doig K, Holt K, Fyfe J, Lavender C, Eddyani M, Portaels F, Yeboah-Manu D, Pluschke G, Seemann T, Stinear TP (2012) On the origin of Mycobacterium ulcerans, the causative agent of Buruli ulcer. BMC Genomics 13:258 
9. De Gentile PL, Mahaza C, Rolland F, Carbonnelle B, Verret JL, Chabasse D (1992) Cutaneous ulcer from Mycobacterium ulcerans. A propos of 1 case in French Guiana. Bull Soc Pathol Exot 85:212-214

10. Ablordey A, Hilty M, Stragier P, Swings J, Portaels F (2005) Comparative nucleotide sequence analysis of polymorphic variable-number tandem-repeat loci in Mycobacterium ulcerans. J Clin Microbiol 43:5281-5284

11. Reynaud Y, Millet J, Couvin D, Rastogi N, Brown C, Couppié P, Legrand E (2015) Heterogeneity among Mycobacterium ulcerans from French Guiana revealed by multilocus variable number tandem repeat analysis (MLVA). PLoS One 10:e0118597

12. Morris A, Gozlan RE, Marion E, Marsollier L, Andreou D, Sanhueza D, Ruffine R, Couppié P, Guégan JF (2014) First detection of Mycobacterium ulcerans DNA in environmental samples from South America. PLoS Negl Trop Dis 8:e2660

13. Morris AL, Guégan JF, Andreou D, Marsollier L, Carolan K, Le Croller M, Sanhueza D, Gozlan RE (2016) Deforestation-driven food-web collapse linked to emerging tropical infectious disease, Mycobacterium ulcerans. Sci Adv 2:e1600387

Open Access This chapter is licensed under the terms of the Creative Commons Attribution 4.0 International License (http://creativecommons.org/licenses/by/4.0/), which permits use, sharing, adaptation, distribution and reproduction in any medium or format, as long as you give appropriate credit to the original author(s) and the source, provide a link to the Creative Commons license and indicate if changes were made.

The images or other third party material in this chapter are included in the chapter's Creative Commons license, unless indicated otherwise in a credit line to the material. If material is not included in the chapter's Creative Commons license and your intended use is not permitted by statutory regulation or exceeds the permitted use, you will need to obtain permission directly from the copyright holder.

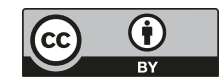

